\title{
Flexibility of Embeddings of Bouquets of Circles on the Projective Plane and Klein Bottle*
}

\author{
Yan Yang and Yanpei Liu \\ Department of Mathematics \\ Beijing Jiaotong University, Beijing, P.R.China \\ yanyang0206@126.com, ypliu@bjtu.edu.cn
}

Submitted: Oct 9, 2007; Accepted: Nov 15, 2007; Published: Nov 23, 2007

Mathematics Subject Classifications: 05C10, 05C30

\begin{abstract}
In this paper, we study the flexibility of embeddings of bouquets of circles on the projective plane and the Klein bottle. The numbers (of equivalence classes) of embeddings of bouquets of circles on these two nonorientable surfaces are obtained in explicit expressions. As their applications, the numbers (of isomorphism classes) of rooted one-vertex maps on these two nonorientable surfaces are deduced.
\end{abstract}

\section{Introduction}

A surface is a compact 2-dimensional manifold without boundary. It can be represented by a polygon of even edges in the plane whose edges are pairwise identified and directed clockwise or counterclockwise. Such polygonal representations of surfaces can be also written by words. For example, the sphere is written as $O_{0}=a a^{-}$where $a^{-}$is paired with a, but with the opposite direction of $a$ on the boundary of the polygon. The projective plane, the torus and the Klein bottle are represented respectively by $a a, a b a^{-} b^{-}$and $a a b b$. In general, $O_{p}=\prod_{i=1}^{p} a_{i} b_{i} a_{i}^{-} b_{i}^{-}$and $N_{q}=\prod_{i=1}^{q} a_{i} a_{i}$ denote, respectively, a surface of orientable genus $p$ and a surface of nonorientable genus $q$. Of course, $N_{1}, O_{1}$ and $N_{2}$ are, respectively, the projective plane, the torus and the Klein bottle. Every surface is homeomorphic to precisely one of the surface $O_{p}(p \geq 0)$, or $N_{q}(q \geq 1)[9,14]$. Suppose $A=a_{1} a_{2} \cdots a_{t}, t \geq 1$, is a word, then $A^{-}=a_{t}^{-} \cdots a_{2}^{-} a_{1}^{-}$is called the inverse of $A$.

Let $\mathcal{S}$ be the collection of surfaces and let $A B$ be a surface. The following topological transformations and their inverses do not change the orientability and genus of a surface:

TT 1: $A a a^{-} B \Leftrightarrow A B$ where $a \notin A B, \quad$ TT 2: $A a b B a b \Leftrightarrow A c B c$ where $c \notin A B$ and

${ }^{*}$ Supported by NNSF of China under Grant No.10571013 
TT 3: $A B \Leftrightarrow(A a)\left(a^{-} B\right)$ where $A B \neq \emptyset$.

Notice that $A$ and $B$ are both linear orders of letters and permitted to be empty. The parentheses stand for cyclic order when more than one cyclic orders occur, for distinguishing from one to another. In fact, what is determined under these operations is just a topological equivalence $\sim$ on $\mathcal{S}$.

The following relations can be deduced by using TT 1-3, as shown in, e.g., [9].

Relation 1: $\left(A x B y C x^{-} D y^{-}\right) \sim\left((A D C B)\left(x y x^{-} y^{-}\right)\right)$,

Relation 2: $(A x B x) \sim\left(\left(A B^{-}\right)(x x)\right)$,

Relation 3: $\left(A x x y z y^{-} z^{-}\right) \sim((A)(x x)(y y)(z z))$.

In these three relations, $A, B, C$, and $D$ are all linear orders of letters and permitted to be empty. Parentheses are always omitted when unnecessary to distinguish cyclic or linear order.

An embedding of a graph $G$ into a surface $S$ is a homeomorphism $h: G \rightarrow S$ of $G$ into $S$ such that every component of $S-h(G)$ is a 2-cell. Two embeddings $h: G \rightarrow S$ and $g: G \rightarrow S$ of $G$ into a surface $S$ are said to be equivalent if there is a homeomorphism $f: S \rightarrow S$ such that $f \circ h=g$.

Given a graph $G$, how many nonequivalent embeddings of $G$ are there into a given surface. This is one of important problems in embedding flexibility, i.e, the classification problem [16]. Research on this problem can be tracked back to [3] and some results have been obtained, such as [1-2,4-8,15-18,20-21,23] etc.

In the following, we will introduce the joint tree model of a graph embedding, which was established in [9] by Liu, based on his initial work in [10]. Some works have been done based on the joint tree method [5,20-21,23].

Given a spanning tree $T$ of a graph $G$, for $1 \leq i \leq \beta$, we split each cotree edge $e_{i}$ into two semi-edges and label them by the same letter as $a_{i}$ where $\beta$ is the betti number of $G$. The resulting graph is a tree consisting of tree edges in $T$ and $2 \beta$ semi-edges. We denote this new tree by $\hat{T}$. Then indexing the $2 \beta$ semi-edges of $\hat{T}$ by + (always omitted) or - , so that the indices of each pair of semi-edges labelled with same letter can be the same or distinct. A rotation at a vertex $v$, denoted by $\sigma_{v}$, is a cyclic permutation of edges incident with $v$. Let $\sigma_{G}=\prod_{v \in V(G)} \sigma_{v}$ be a rotation system of $G$.

The tree $\hat{T}$ with an index of each semi-edge and a rotation system of $G$ is called a joint tree of $G$. Denote by $\hat{T}_{\sigma}^{\delta}$ the joint tree, in which $\delta=\left(\delta_{1}, \delta_{2}, \cdots, \delta_{\beta}\right)$ be a binary vector, $\delta_{i}$ can be 0 or 1 where $\delta_{i}=0$ means that the two indices of $a_{i}$ are distinct; otherwise, the same. In fact, the edge $a_{i}$ with $\delta_{i}=1$ are the twisted edge [7] in the embedding. By reading these lettered semi-edges with indices of a $\hat{T}_{\sigma}^{\delta}$ in a fixed orientation (clockwise or counterclockwise), we can get an algebraic representation for a surface. It is a cyclic order of $2 \beta$ letters with indices. Such a surface is called an associated surface [11] of $G$. If two associate surfaces of $G$ have the same cyclic order with the same $\delta$ in their algebraic representations, then we say that they are the same; otherwise, distinct.

From [11], there is a 1-to-1 correspondence between associate surfaces and embeddings of a graph, hence an embedding of a graph on a surface can be represented by an associate surface of it. 
Let $G$ be a graph, $g_{p}(G), \tilde{g}_{q}(G), p \geq 0, q \geq 1$ be the numbers (of equivalence classes) of embeddings of $G$ on the surface of orientable genus $p$ and nonorientable genus $q$, respectively.

Denote the bouquet of $n$ circle by $B_{n}$. Gross et al obtain the genus distribution of $B_{n}$ [4], Kwak and Lee obtained the total embedding polynomial of $B_{n+1}$ from that of $B_{n}$ inductively and derive the total genus distribution of $B_{n+1}$ from it in [7], but we can't get the numbers of embeddings of $B_{n}$ on nonorientable surfaces from their results easily and directly. In this paper, by joint tree model, we study the flexibility of embeddings of $B_{n}$ on the projective plane and the Klein bottle. The numbers of embeddings of $B_{n}$ on these two nonorientable surface are obtained in explicit expressions. Then the structures of those embeddings are described. As their applications, the numbers (of isomorphism classes) of rooted one-vertex maps on these two nonorientable surfaces are deduced.

\section{Some Lemmas}

Lemma 2.1[11] An orientable surface $S$ is a surface of orientable genus 0 if and only if there is no form as $A x B y C x^{-1} D y^{-1}$ in it.

Lemma 2.2 Let $S$ be a nonorientable surface, if there is a form as $A x B y C x^{-} D y^{-}$in $S$, then the genus of $S$ will be not less than 3 ; if there is a form as $A x B y C x^{-} D y$ or $A x B y C y D x$ in $S$, then the genus of $S$ will be not less than 2 .

Proof: If the form as $A x B y C x^{-} D y^{-}$exists in $S$, by Relation $1, A x B y C x^{-} D y^{-}$ $\sim A D C B x y x^{-} y^{-}$, and there is at least one pair of semi-edges $z, z^{\varepsilon}$ with same indices, because $S$ is nonorientable. By Relation 1-3, we can get $S \sim A^{\prime} z z x y x^{-} y^{-} \sim A^{\prime} z z x x y y$. So the genus will be not less than 3 .

If the form as $A x B y C x^{-} D y$ exists, by using Relation 2 twice, we get that $A x B y C x^{-} D y \sim A x B D^{-} x C^{-} y y \sim A D B^{-} C^{-} x x y y$, so the genus of $S$ will be not less than 2. In the same way, we can get that the genus of $S$ where the form $A x B y C y D x$ exists will be not less than 2 . Thus the proof is complete.

The following lemma can be got from both [4] and [9] easily.

Lemma 2.3[4,9] The number of embeddings of $B_{n}$ on the sphere is

$$
g_{0}\left(B_{n}\right)=(n-1) ! 2^{n-1} \frac{\left(\begin{array}{c}
2 n \\
n
\end{array}\right)}{n+1} .
$$

The Catalan sequence is the sequence $C_{0}, C_{1}, C_{2}, \ldots, C_{n}, \ldots$ where $C_{n}=\frac{\left(\begin{array}{c}2 n \\ n\end{array}\right)}{n+1}(n=$ $0,1,2, \ldots)$ is the nth Catalan number. Let $F(x)=\sum_{n \geq 0} C_{n} x^{n}$ be the generating function of $\left\{C_{n}\right\}_{n \geq 0}$, then $F(x)=\frac{1-\sqrt{1-4 x}}{2 x}[19]$. 
Lemma 2.4 For integers $n \geq k \geq 0$, we have

$$
\sum_{\substack{x_{1}+\cdots+x_{2 k}=n-k \\
x_{j} \geq 0,1 \leq j \leq 2 k}} \prod_{j=1}^{2 k} \frac{\left(\begin{array}{c}
2 x_{j} \\
x_{j}
\end{array}\right)}{\left(x_{j}+1\right)}=\frac{2 k(2 n-1) !}{(n-k) !(n+k) !}
$$

Proof: For $F(x)=\frac{1-\sqrt{1-4 x}}{2 x}=\sum_{n=0}^{\infty} C_{n} x^{n}$, from [19],

$$
F^{m}(x)=\sum_{n=0}^{\infty} \frac{m(2 n+m-1) !}{n !(n+m) !} x^{n}, m \geq 1,
$$

and

$$
F^{m}(x)=\left(\sum_{n=0}^{\infty} C_{n} x^{n}\right)^{m}=\sum_{n=0}^{\infty}\left(\sum_{\substack{x_{1}+\cdots+x_{m}=n \\ x_{j} \geq 0,1 \leq j \leq m}} \prod_{j=1}^{m} C_{x_{j}}\right) x^{n}
$$

from (1) and (2), we can get that

$$
\sum_{\substack{x_{1}+\cdots+x_{m}=n \\ x_{j} \geq 0,1 \leq j \leq m}} \prod_{j=1}^{m} C_{x_{j}}=\frac{m(2 n+m-1) !}{n !(n+m) !} .
$$

Let $m=2 k, n=n-k$, the lemma follows.

\section{The number of embeddings of bouquets of circles on the projective plane}

Let $S$ be a surface. If $x, y \in S$ are in the form as $S=A x B y C x D y$, then they are said to be interlaced; otherwise, parallel.

Theorem 3.1 All the associate surfaces of $B_{n}$ on the projective plane have the form as

$$
A_{1} a_{1} \cdots A_{k} a_{k} A_{k+1} a_{1} \cdots A_{2 k} a_{k}, 1 \leq k \leq n
$$

in which $A_{i}$ is either empty or $A_{i} \sim O_{0}, 1 \leq i \leq 2 k$.

Proof: Suppose that there are $k(1 \leq k \leq n)$ twisted edges $a_{1}, \ldots, a_{k}$ in the embedding of $B_{n}$ on the projective plane. When $k \geq 2$, each pair of $a_{i}, a_{j}, i \neq j$ must be interlaced in the associate surface, otherwise its nonorientable genus will be greater than one, according to Relation 2. So the associate surfaces have the form as $A_{1} a_{1} \cdots A_{k} a_{k} A_{k+1} a_{1} \cdots A_{2 k} a_{k}$, it still holds when $k=1$. For $2(n-k)$ semi-edges corresponding to the $n-k$ untwisted edges in $A_{i}, 1 \leq i \leq 2 k$, we have $\forall x \in A_{i}, x^{-} \in A_{i}$, by Lemma 2.2. And according to Relation 2,

$$
A_{1} a_{1} \cdots A_{k} a_{k} A_{k+1} a_{1} \cdots A_{2 k} a_{k} \sim A_{1} A_{k+1}^{-} a_{k}^{-} A_{k}^{-} \cdots a_{2}^{-} A_{2}^{-} A_{k+2} a_{2} \cdots A_{2 k} a_{k} a_{1} a_{1} .
$$




$$
\begin{aligned}
& A_{1} a_{1} \cdots A_{k} a_{k} A_{k+1} a_{1} \cdots A_{k+k} a_{k} \sim N_{1} \\
\Leftrightarrow & A_{1} A_{k+1}^{-} a_{k}^{-} A_{k}^{-} \cdots a_{2}^{-} A_{2}^{-} A_{k+2} a_{2} \cdots A_{2 k} a_{k} \sim O_{0} \\
\Leftrightarrow & A_{i} \sim O_{0}, 1 \leq i \leq 2 k .
\end{aligned}
$$

Thus the theorem is obtained.

The structure of the embeddings of $B_{n}$ on the projective plane is shown in Fig.1.

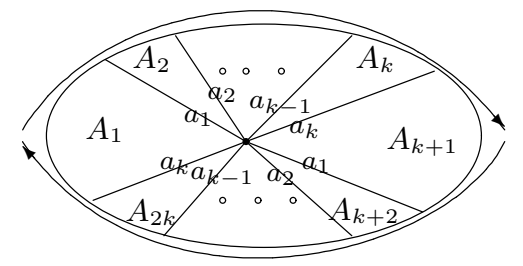

Fig.1 $B_{n}$ on the projective plane

Theorem 3.2 The number of the embeddings of $B_{n}$ on the projective plane is

$$
\tilde{g}_{1}\left(B_{n}\right)=(n-1) ! 2^{n-1}\left(2^{2 n-1}-\frac{(2 n) !}{2(n !)^{2}}\right) .
$$

Proof: By the joint tree method, there are $\left(\begin{array}{l}n \\ k\end{array}\right)(k-1) ! 2^{k-1}$ ways to choose and place the $k, 1 \leq k \leq n$ twisted edges, corresponding to $2 k$ semi-edges $a_{1}, a_{1}, \ldots, a_{k}, a_{k}$, in the associate surface. Suppose that there are $2 x_{j}$ semi-edges in $A_{j}, 1 \leq j \leq 2 k$, then each of the $2 x_{j}$ semi-edges can be the first semi-edge in $A_{j}$, i.e., each of the $2 x_{j}$ letters can be the first letter in the liner order of letters $A_{j}$. According to Theorem 3.1 and Lemmas 2.3, 2.4 , the number of ways to put $2(n-k)$ semi-edges, corresponding to $n-k$ untwisted edges, into $A_{1}, \ldots, A_{2 k}$ is

$$
\begin{aligned}
& \sum_{\substack{x_{1}+\cdots+x_{2 k}=n-k \\
x_{j} \geq 0,1 \leq j \leq 2 k}} \frac{(n-k) !}{x_{1} ! x_{2} ! \cdots x_{2 k} !} \prod_{j=1}^{2 k}\left(2 x_{j} g_{0}\left(B_{x_{j}}\right)\right) \\
= & 2^{n-k}(n-k) ! \sum_{\substack{x_{1}+\cdots+x_{2 k}=n-k \\
x_{j} \geq 0,1 \leq j \leq 2 k}} \prod_{j=1}^{2 k} \frac{\left(\begin{array}{l}
2 x_{j} \\
x_{j}
\end{array}\right)}{\left(x_{j}+1\right)} \\
= & \frac{2^{n-k+1} k(2 n-1) !}{(n+k) !} .
\end{aligned}
$$

For $1 \leq k \leq n$, the number of embeddings of $B_{n}$ on the projective plane is

$$
\sum_{k=1}^{n}\left(\begin{array}{l}
n \\
k
\end{array}\right)(k-1) ! 2^{k-1} \frac{2^{n-k+1} k(2 n-1) !}{(n+k) !}=(n-1) ! 2^{n-1} \sum_{k=1}^{n}\left(\begin{array}{c}
2 n \\
n-k
\end{array}\right),
$$

by simplification, the theorem is obtained. 


\section{The number of embeddings of bouquets of circles on the Klein bottle}

There are at least 4 edges in the polygonal representations of the Klein bottle. And there are only two ways, i.e., $a a b b$ and $a b a b^{-}$. From those words, the associate surfaces of $B_{n}$

on the Klein bottle can be classified into two cases, according to the places of the twisted edges. Case 1, all the twisted edges are of the form

$$
A_{1} a_{1} \cdots A_{i} a_{i} A_{i+1} a_{1} \cdots A_{2 i} a_{i} D_{1} b_{1} \cdots D_{j} b_{j} D_{j+1} b_{1} \cdots D_{2 j} b_{j}, i, j \geq 1, i+j \leq n
$$

Case 2, all the twisted edges are of the form

$$
A_{1} a_{1} \cdots A_{k} a_{k} A_{k+1} a_{1} \cdots A_{2 k} a_{k}, 1 \leq k \leq n-1
$$

in which $A_{1}, \ldots, A_{2 i}, D_{1}, \ldots, D_{2 j}$ can be empty.

Theorem 4.1 The associate surfaces in Case 1 are Klein bottles if and only if one of the following two conditions holds:

(1) $A_{1} D_{1} \sim O_{0}, A_{t} \sim D_{k} \sim O_{0}, t \in[2,2 i], k \in[2,2 j]$;

(2) $A_{i+1} D_{j+1} \sim O_{0}, A_{t} \sim D_{k} \sim O_{0}, t \in[1, i] \cup[i+2,2 i], k \in[1, j] \cup[j+2,2 j]$.

Proof: $\quad$ By using Relation 2 twice,

$$
\begin{aligned}
& A_{1} a_{1} \cdots A_{i} a_{i} A_{i+1} a_{1} \cdots A_{2 i} a_{i} D_{1} b_{1} \cdots D_{j} b_{j} D_{j+1} b_{1} \cdots D_{2 j} b_{j} \\
& \sim A_{1} a_{1} \cdots A_{i-1} a_{i-1} A_{i} A_{2 i}^{-1} a_{i-1}^{-1} A_{2 i-1}^{-1} \cdots a_{1}^{-1} A_{i+1}^{-1} \\
& D_{1} b_{1} \cdots D_{j-1} b_{j-1} D_{j} D_{2 j}^{-1} b_{j-1}^{-1} D_{2 j-1}^{-1} \cdots b_{1}^{-1} D_{j+1}^{-1} a_{i} a_{i} b_{j} b_{j} \\
&(*) \sim N_{2} \Leftrightarrow A_{1} a_{1} \cdots A_{i-1} a_{i-1} A_{i} A_{2 i}^{-1} a_{i-1}^{-1} A_{2 i-1}^{-1} \cdots a_{1}^{-1} A_{i+1}^{-1} \\
& D_{1} b_{1} \cdots D_{j-1} b_{j-1} D_{j} D_{2 j}^{-1} b_{j-1}^{-1} D_{2 j-1}^{-1} \cdots b_{1}^{-1} D_{j+1}^{-1} \sim O_{0} \\
& \Leftrightarrow A_{i} A_{2 i}^{-1} \sim \cdots \sim A_{2} A_{i+2}^{-1} \sim D_{j} D_{2 j}^{-1} \sim \cdots \sim D_{2} D_{j+2}^{-1} \\
& \sim A_{1} A_{i+1}^{-1} D_{1} D_{j+1}^{-1} \sim O_{0}
\end{aligned}
$$

Because the semi-edges in $A_{t}, B_{k}, 1 \leq t \leq 2 i, 1 \leq k \leq 2 j$, are all corresponding to untwisted edges, according to Lemma 2.1,

$$
\begin{gathered}
\forall x \in A_{t}, x^{-1} \in A_{t}, \quad t \neq 1, i+1, \quad \forall y \in D_{k}, y^{-1} \in D_{k}, k \neq 1, j+1 . \\
A_{t} \sim D_{k} \sim O_{0}, \quad t \neq 1, i+1, k \neq 1, j+1 .
\end{gathered}
$$

For the same reason,

$$
\forall x \in A_{1}, x^{-1} \in A_{1} \text { or } x^{-1} \in D_{1} ; \quad \forall y \in A_{i+1}, y^{-1} \in A_{i+1} \text { or } y^{-1} \in D_{j+1} \text {. }
$$

If $\forall x \in A_{1}, x^{-1} \in A_{1}$ and $\forall y \in D_{1}, y^{-1} \in D_{1}$, then

$$
A_{1} A_{i+1}^{-1} D_{1} D_{j+1}^{-1} \sim O_{0} \Leftrightarrow A_{1} \sim D_{1} \sim O_{0}, A_{i+1} D_{j+1} \sim O_{0} .
$$


The condition 2 holds.

If $\exists x \in A_{1}, x^{-1} \in D_{1}$, or $\exists y \in D_{1}, y^{-1} \in A_{1}$, then $\forall x \in A_{i+1}, x^{-1} \in A_{i+1}, \forall y \in D_{j+1}$, $y^{-1} \in D_{j+1}$, according to Lemma 2.1. Hence we have that

$$
A_{1} A_{i+1}^{-1} D_{1} D_{j+1}^{-1} \sim O_{0} \Leftrightarrow A_{1} D_{1} \sim O_{0}, A_{i+1} \sim D_{j+1} \sim O_{0}
$$

The condition 1 holds. If $A_{1}=\emptyset$, then $D_{1} \sim A_{i+1}^{-} D_{j+1}^{-} \sim O_{0}$, the condition 2 holds. Above all, we get the conclusion.

Theorem 4.2 The associate surfaces in Case 2 are Klein bottles if and only if one of the following $k$ conditions holds: $A_{i} A_{k+i}^{-1} \sim N_{1}, A_{j} \sim O_{0}, j \neq i, i+k, 1 \leq i \leq k$.

Proof: By Relation 2,

$$
\begin{aligned}
& A_{1} a_{1} \cdots A_{k} a_{k} A_{k+1} a_{1} \cdots A_{k+k} a_{k} \sim N_{2} \\
\Leftrightarrow & A_{1} a_{1} \cdots A_{k-1} a_{k-1} A_{k} A_{2 k}^{-1} a_{k-1}^{-1} A_{2 k-1}^{-1} \cdots a_{1}^{-1} A_{k+1}^{-1} \sim N_{1},
\end{aligned}
$$

according to Lemma 2.2, the theorem follows.

The structures of the embeddings of $B_{n}$ on the Klein bottle in Cases 1 and 2 are shown in Fig.2 and Fig3, respectively. Fig.2 describes the condition (1) in Theorem 4.1, Fig.3 describes the condition $A_{1} A_{k+1}^{-} \sim N_{1}, A_{j} \sim O_{0}, j \neq 1,1+k$, in Theorem 4.2.

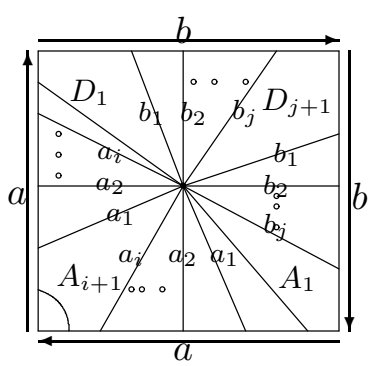

Fig.2 $B_{n}$ on the Klein bottle in Case 1

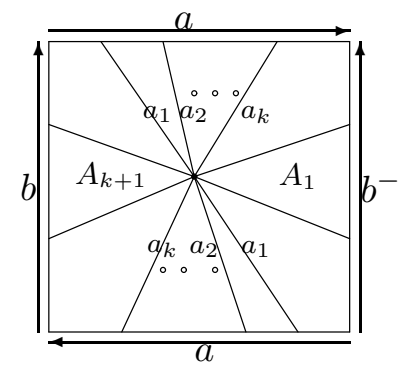

Fig. $3 B_{n}$ on the Klein bottle in Case 2

For convenience, we write $\sum_{\substack{x_{1}+\cdots+x_{2 k}=n-k \\ x_{j} \geq 0,1 \leq j \leq 2 k}} \prod_{j=1}^{2 k} \frac{\left(\begin{array}{c}2 x_{j} \\ x_{j}\end{array}\right)}{\left(x_{j}+1\right)}$ as $G(n, k)$ in the following.

Theorem 4.3 The number of embeddings of $B_{n}$ on the Klein bottle in Case 1 is

$$
\tilde{g}_{2}^{1}\left(B_{n}\right)=(n-1) ! 2^{n-1} \sum_{t=1}^{n} t(t-1)^{2}\left(\begin{array}{c}
2 n \\
n-t
\end{array}\right) .
$$

Proof: For the symmetry, we suppose that $i \leq j$. For the convenience of calculation, we subdivide the two conditions in Theorem 4.1 into there conditions as follows:

a) $A_{t} \sim D_{k} \sim O_{0}, 1 \leq t \leq 2 i, 1 \leq k \leq 2 j$. 
b) $A_{1} D_{1} \sim O_{0}, A_{t} \sim D_{k} \sim O_{0}, t, k \neq 1$.

c) $A_{i+1} D_{j+1} \sim O_{0}, A_{t} \sim D_{k} \sim O_{0}, t \neq i+1, k \neq j+1$.

We discuss the three conditions respectively, as follows.

a) If $j>i$, the number of ways to choose and place $b_{1}, \cdots, b_{j}$ is $\left(\begin{array}{c}n \\ j\end{array}\right)(j-1) ! 2^{j-1}$, then the number of ways to choose and place $a_{1}, \cdots, a_{i}$ is $\left(\begin{array}{c}n-j \\ i\end{array}\right) i ! 2^{i} 2 j$, similar with the argument in the proof of Theorem 3.2, the number of ways of embeddings of the $n-i-j$ untwisted edges is $2^{n-j-i}(n-j-i) ! G(n, j+i)$. Hence, for the given $i$ and $j$, the number of embeddings is $2^{n}\left(\begin{array}{c}n \\ j\end{array}\right)(j-1) !\left(\begin{array}{c}n-j \\ i\end{array}\right) i ! j(n-j-i) ! G(n, j+i)=n ! 2^{n} G(n, j+i)$.

For $1 \leq i \leq\left\lfloor\frac{n}{2}\right\rfloor, i+1 \leq j \leq n-i$, the number of embeddings of $B_{n}$ on the Klein bottle is

$$
n ! 2^{n} \sum_{i=1}^{\left\lfloor\frac{n}{2}\right\rfloor} \sum_{j=i+1}^{n-i} G(n, j+i)=n ! 2^{n} \sum_{t=3}^{n}\left(\left\lceil\frac{t}{2}\right\rceil-1\right) G(n, t) .
$$

If $j=i$, for the symmetry, the number of embeddings is $\frac{1}{2} n ! 2^{n} \sum_{i=1}^{\left\lfloor\frac{n}{2}\right\rfloor} G(n, 2 i)$.

So the number of embeddings of $B_{n}$ on the Klein bottle in condition $a$ ) is

$$
\begin{aligned}
\tilde{g}_{2}^{1 a}\left(B_{n}\right) & =n ! 2^{n}\left(\sum_{t=3}^{n}\left(\left\lceil\frac{t}{2}\right\rceil-1\right) G(n, t)+\frac{1}{2} \sum_{i=1}^{\left\lfloor\frac{n}{2}\right\rfloor} G(n, 2 i)\right) \\
& =n ! 2^{n-1} \sum_{t=2}^{n}(t-1) G(n, t) .
\end{aligned}
$$

b) Let $M=\left\{r \mid r \in A_{1}, r^{-1} \in D_{1}\right\}, m=|M| \geq 1$. The argument is similar with that of condition $a$ ). If $j>i$, the number of embeddings is

$$
\begin{aligned}
& n ! 2^{n} \sum_{i=1}^{\left\lfloor\frac{n}{2}\right\rfloor} \sum_{j=i+1}^{n-i-1} \sum_{m=1}^{n-j-i} G(n, j+i+m) \\
= & n ! 2^{n} \sum_{t=4}^{n} \sum_{k=3}^{t-1}\left(\left\lceil\frac{k}{2}\right\rceil-t-3\right) G(n, t) \\
= & n ! 2^{n} \sum_{t=4}^{n}\left(\left\lceil\frac{t}{2}\right\rceil-1\right)\left(\left\lfloor\frac{t}{2}\right\rfloor-1\right) G(n, t) .
\end{aligned}
$$

If $j=i$, the number of embeddings is

$$
\frac{1}{2} n ! 2^{n} \sum_{i=1}^{\left\lfloor\frac{n}{2}\right\rfloor} \sum_{m=1}^{n-2 i} G(n, 2 i+m)=\frac{1}{2} n ! 2^{n} \sum_{t=3}^{n}\left(\left\lceil\frac{t}{2}\right\rceil-1\right) G(n, t) .
$$


So the number of embeddings of $B_{n}$ on the Klein bottle in condition $b$ ) is

$$
\begin{aligned}
\tilde{g}_{2}^{1 b}\left(B_{n}\right) & =n ! 2^{n} \sum_{t=3}^{n}\left(\left(\left\lceil\frac{t}{2}\right\rceil-1\right)\left(\left\lfloor\frac{t}{2}\right\rfloor-1\right)+\frac{1}{2}\left(\left\lceil\frac{t}{2}\right\rceil-1\right)\right) G(n, t) \\
& =n ! 2^{n-2} \sum_{t=3}^{n}(t-1)(t-2) G(n, t) .
\end{aligned}
$$

For the symmetry, the number of embeddings of $B_{n}$ on the Klein bottle in condition $c$ ) is $\tilde{g}_{2}^{1 c}\left(B_{n}\right)=\tilde{g}_{2}^{1 b}\left(B_{n}\right)$.

So the number of embeddings of $B_{n}$ on the Klein bottle in Case 1 is

$$
\begin{aligned}
\tilde{g}_{2}^{1}\left(B_{n}\right) & =\tilde{g}_{2}^{1 a}\left(B_{n}\right)+\tilde{g}_{2}^{1 b}\left(B_{n}\right)+\tilde{g}_{2}^{1 c}\left(B_{n}\right) \\
& =n ! 2^{n-1} \sum_{t=1}^{n}(t-1)^{2} G(n, t)=(n-1) ! 2^{n-1} \sum_{t=1}^{n} t(t-1)^{2}\left(\begin{array}{c}
2 n \\
n-t
\end{array}\right),
\end{aligned}
$$

the theorem is obtained.

Theorem 4.4 The number of embeddings of $B_{n}$ on the Klein bottle in Case 2 is

$$
\tilde{g}_{2}^{2}\left(B_{n}\right)=(n-1) ! 2^{n-1} \sum_{t=1}^{n} t(t-1)\left(\begin{array}{c}
2 n \\
n-t
\end{array}\right) .
$$

Proof: The number of ways to choose and place $a_{1}, \cdots, a_{k}$ is $\left(\begin{array}{l}n \\ k\end{array}\right)(k-1) ! 2^{k-1}$, then choose one of the $k$ conditions in Theorem 4.2. Suppose we choose $A_{k} A_{2 k}^{-1} \sim N_{1}$, then $A_{k} A_{2 k}^{-1}$ has the form as $\tilde{A}_{1} t_{1} \cdots \tilde{A}_{|\Gamma|} t_{|\Gamma|} \tilde{A}_{|\Gamma|+1} t_{1} \cdots \tilde{A}_{2|\Gamma|} t_{|\Gamma|}$, according to Theorem 3.1, in which $\Gamma=\left\{t \mid t \in A_{k}\right.$ and $\left.t^{-1} \in A_{2 k}\right\}$. Let $m=|\Gamma|$, then $1 \leq m \leq n-k$, the number of ways to place the $m$ untwisted edges is $\left(\begin{array}{c}n-k \\ m\end{array}\right) m ! 2^{m}$. And the number of ways to put the $2(n-k-m)$ semi-edges, corresponding to the left $n-k-m$ untwisted edges, into $A_{j}, \tilde{A}_{i}$ such that $A_{j} \sim \tilde{A}_{i} \sim O_{0}, j \in[1, k-1] \cup[k+1,2 k-1], 1 \leq i \leq 2 m$ is $2^{n-k-m}(n-k-m) ! G(n, k+m)$. Hence, the number of embeddings is

$n ! 2^{n-1} \sum_{k=1}^{n-1} \sum_{m=1}^{n-k} G(n, k+m)=n ! 2^{n-1} \sum_{t=2}^{n}(t-1) G(n, t)=(n-1) ! 2^{n-1} \sum_{t=1}^{n} t(t-1)\left(\begin{array}{c}2 n \\ n-t\end{array}\right)$,

the theorem is obtained.

Theorem 4.5 The number of embeddings of $B_{n}$ on the Klein bottle is

$$
\tilde{g}_{2}\left(B_{n}\right)=n ! 2^{n-1}\left(\frac{(2 n-1) !}{((n-1) !)^{2}}-4^{n-1}\right)
$$


Proof: The number of embeddings of $B_{n}$ on the Klein bottle is $\tilde{g}_{2}\left(B_{n}\right)=\tilde{g}_{2}^{1}\left(B_{n}\right)+\tilde{g}_{2}^{2}\left(B_{n}\right)$, according to Theorems 4.3, 4.4, $\tilde{g}_{2}\left(B_{n}\right)=(n-1) ! 2^{n-1} \sum_{t=1}^{n} t^{2}(t-1)\left(\begin{array}{c}2 n \\ n-t\end{array}\right)$. By calculation and certain simplification, one can get that

$$
\sum_{t=1}^{n} t^{2}(t-1)\left(\begin{array}{c}
2 n \\
n-t
\end{array}\right)=n\left(\frac{(2 n-1) !}{((n-1) !)^{2}}-4^{n-1}\right)
$$

thus the theorem follows.

\section{Examples}

According to Theorems 3.2, 4.5, one can calculate the numbers of embeddings of $B_{n}$ on the projective plane and Klein bottle easily, give these numbers $\tilde{g}_{1}\left(B_{n}\right), \tilde{g}_{2}\left(B_{n}\right), 1 \leq n \leq 8$ as follows:

\begin{tabular}{|c|c|c|c|c|c|c|c|c|}
\hline & $B_{1}$ & $B_{2}$ & $B_{3}$ & $B_{4}$ & $B_{5}$ & $B_{6}$ & $B_{7}$ & $B_{8}$ \\
\hline$\tilde{g}_{1}\left(B_{n}\right)$ & 1 & 10 & 176 & 4464 & 148224 & 6090240 & 298414080 & 16987944960 \\
\hline$\tilde{g}_{2}\left(B_{n}\right)$ & 0 & 8 & 336 & 14592 & 718080 & 40273920 & 2553384960 & 181129052160 \\
\hline
\end{tabular}

Note that the numbers in the table are coincident with those in Table 1 given in [7] by Kwak and Lee.

\section{$5 \quad$ Applications}

A map is an embedding of a graph on a surface, the graph is called the underlying graph of the map. The enumeration of maps on surfaces have been developed and deepened by people, based on the initial works by W.T.Tutte in the 1960s. The reader is referred to the monograph [12] for further background about enumerative theory of maps. For a given graph $\Gamma$, the relations between its genus distribution of rooted maps and genus distribution of embeddings on orientable and nonorientable surfaces are given in [13]. We adopt the same notations as that in [13], where $r[\Gamma](x)(\tilde{r}[\Gamma](x))$ and $g[\Gamma](x)(\tilde{g}[\Gamma](x))$ denote rooted orientable (nonorientable) map polynomial on genus and orientable (nonorientable) genus polynomial of $\Gamma$, respectively.

Theorem 5.1[13] For a connected graph $\Gamma$

$$
\left|A u t_{\frac{1}{2}} \Gamma\right| r[\Gamma](x)=2 \varepsilon(\Gamma) g[\Gamma](x)
$$

and

$$
\left|A u t_{\frac{1}{2}} \Gamma\right| \tilde{r}[\Gamma](x)=2 \varepsilon(\Gamma) \tilde{g}[\Gamma](x)
$$

where $A u t_{\frac{1}{2}} \Gamma$ and $\varepsilon(\Gamma)$ denote the semi-arc automorphism group and the size of $\Gamma$. 
From [13], $\left|A u t_{\frac{1}{2}} B_{n}\right|=2^{n} n$ !, by Theorems 3.2, 4.5 and 5.1, one can get the number (of isomorphism classes) $\tilde{r}_{i}\left(B_{n}\right)$ of rooted maps with the underlying graph $B_{n}$ on the nonorientable surface of genus $i(i=1,2)$.

Corollary 5.1 The number of rooted maps with the underlying graph $B_{n}$ on the projective plane is

$$
\tilde{r}_{1}\left(B_{n}\right)=2^{2 n-1}-\frac{(2 n) !}{2(n !)^{2}}
$$

Corollary 5.2 The number of rooted maps with the underlying graph $B_{n}$ on the Klein bottle is

$$
\tilde{r}_{2}\left(B_{n}\right)=n\left(\frac{(2 n-1) !}{((n-1) !)^{2}}-4^{n-1}\right) .
$$

The rooted maps with the underlying graph $B_{n}$ is also called one-vertex maps. In [22], the numbers of one-vertex maps on the projective plane and the Klein bottle are also obtained, by enumerating functional equation. The result in Corollary 5.1 is the same to that in [22], and the result in Corollary 5.2 is in much simpler form than that in [22].

\section{References}

[1] J.Chen, J.L.Gross and R.G.Rieper, Overlap matrices and total imbedding distribution, Discrete Math. 128 (1994) 73-94.

[2] M.L.Furst, J.L.Gross and R.Statman, Genus distributions for two classes of graphs, J. Combin. Theory (B) 46 (1989) 22-36.

[3] J.L.Gross and M.L.Furst, Hierarchy of imbedding distribution invariants of graph, J.Graph Theory 11 (1987) 205-220.

[4] J.L.Gross, D.P.Robbins and T.W.Tucker, Genus distributions for bouquets of circles, J. Combin. Theory (B) 47 (1989) 292-306.

[5] R.X.Hao and Y.P.Liu, The genus distributions of directed antiladders in orientable surfaces, Appl.Math.Lett. (2007), doi:10.1016/j.aml.2007.05.001.

[6] J.H.Kwak and J.Lee, Genus polynomials of Dipoles, Kyungpook Math. Journal, 33 (1993) 115-125.

[7] J.H.Kwak and S.H.Shim, Total embedding distributions for bouquets of circles. Discrete Math. 248 (2002) 93-108.

[8] Y.P.Liu, Embeddability in Graphs. Kluwer, Dordrecht/Boston/London, 1995.

[9] Y.P.Liu, Advances in Combinatorial Maps (in Chinese). Beijing: Northern JiaoTong University Press, 2003.

[10] Y.P.Liu, The nonorientable maximum genus of a graph(in Chinese), Scientia Sinica, Special Issue on math I (1979) 191-201. 
[11] Y.P.Liu, Algebraic Principles of Maps (in Chinese). Beijing: Higher Education Press, 2006.

[12] Y.P.Liu, Enumerative Theory of Maps. Kluwer, Dordrecht/Boston/London, 1999.

[13] L.F.Mao, Y.P.Liu and E.L.Wei, The semi-arc automorphism group of a graph with application to map enumeration, Graphs and Combinatorics, 22 (2006) 83-101.

[14] W.S.Massey, Algebraic Topology: An Introduction. Harcourt, Brace and World, New York, 1967.

[15] H.Ren and M.Deng, Flexibility of embeddings of wheel graphs on the torus, J. East China Normal University (Natural Science) 1 (2006) 57-62.

[16] N.Robertson, X.Y.Zha and Y.Zhao, On the flexibility of toroidal embeddings, J. Combin. Theory (B)(2007), doi:10.1016/j.jctb.2007.03.006.

[17] S.Stahl, On the number of maximum genus embeddings of almost all graphs, Europ. J. Combin. 13 (1992) 119-126.

[18] E.H.Tesar, Genus distributions for Ringel ladders, Discrete Math. 216 (2000) 235-252.

[19] H.S.Wilf, Generatingfunctionology (Second Edition). Academic Press, 1994.

[20] L.X.Wan and Y.P.Liu, Orientable embedding distributions by genus for certain type of non-planar graph (I), Ars Combin. 79 (2006) 97-105.

[21] L.X.Wan and Y.P.Liu, Orientable embedding genus distribution for certain types of graphs, J. Combin. Theory (B)(2007), doi:10.1016/j.jctb.2007.04.002.

[22] Y.Xu and Y.P.Liu, Counting pan-fan maps on nonorientable surfaces, Ars Combin. 83 (2007) 15-32.

[23] Y.Yang and Y.P.Liu, Total genus distributions of two classes of 4-regular graphs (in Chinese), Acta Math Sinica (Chinese Series) 50(5) (2007) 1191-1200. 